\title{
Immunologic Studies of Arylsulfatase $A$ in Normal and Metachromatic Leukodystrophy Liver
}

\author{
EMMANUEL SHAPIRA, RON R. DEGREGORIO, AND HENRY L. NADLER ${ }^{(27)}$ \\ Division of Genetics, Children's Memorial Hospital, Department of Pediatrics, Northwestern University Medical \\ School, Chicago, Illinois, USA
}

\section{Summary}

Purified human liver arylsulfatase $\mathbf{A}$ on polyacrylamide gel electrophoresis at $\mathrm{pH} 4.0$ is separated into two protein forms with enzymatic activity and two distinct inactive subunits. All of these components were immunologically distinguishable using different antisera preparations. In Iate infantile metachromatic leukodystrophy, only one of the two inactive subunits was immunologically detected, whereas in the juvenile form of metachromatic leukodystrophy, both inactive subunits were antigenically present.

\section{Speculation}

Arylsulfatase $A$ is a dimeric enzyme composed of two distinct subunits. In late infantile metachromatic leukodystrophy, the molecular defect results in the absence of one of the subunits and therefore no active dimer is formed. In contrast, in the juvenile form of the disease, the mutation does not cause the absence of either of the subunits, as they are immunologically detectable, but does cause the formation of an unstable dimer.

Arylsulfatase A (ASA) (22) (arylsulfate sulfohydrolase A, EC 3.1.6.1.) is a lysosomal enzyme (6) present in various human tissues $(4,7)$. Deficiency of this enzyme has been described in a group of familial metabolic disorders, metachromatic leukodystrophies (MLD) (1). The relationship between the molecular defect in arylsulfatase and its expression in the various clinical forms of MLD is unknown.

ASA from human liver has been purified by relatively simple procedures and partially characterized (19). Purified ASA (mol wt 107,000) was irreversibly converted into inactive subunits with $58,000 \mathrm{~mol}$ wt when subjected to $\mathrm{pH}$ values below 4 or in the presence of $1 \%$ sodium dodecyl sulfate (SDS). This phenomenon of conversion of the active ASA into low molecular weight inactive subunits at acidic $\mathrm{pH}$ or in the presence of SDS is consistent with that described for ox liver ASA $(14,15,17)$. At $\mathrm{pH} 8.5$, purified human ASA migrates as a single band with

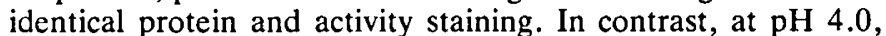
two active molecular forms and two distinct inactive subunits were revealed (19).

Three clinical variants of MLD (late infantile, juvenile, and adult), all transmitted as autosomal recessive disorders, have been described (13). The relationship between the clinical courses suggest different mutational events. Cerebroside-3-sulfate accumulates in all three variants (11). The amount of cerebroside-3-sulfate and the degree of sulfatide accumulation differ in the late infantile MLD and the juvenile $\operatorname{MLD}(8,16)$.

In both the late infantile and juvenile forms of MLD, a protein that is immunologically indistinguishable from normal ASA was described $(11,12,20)$. In a recent publication,
Shapira and Nadler (18) demonstrated that the antigenically cross-reacting material in late infantile and juvenile MLD is enzymatically inactive. The present study utilizing monospecific antisera to ASA and its subunits demonstrates that ASA is composed of two immunologically distinct subunits. Different antigenic determinants of the subunits were expressed when in the active dimer form or in the inactive denatured subunit form. In late infantile MLD, one of these subunits was absent. In the juvenile form of the disease, both inactive subunits were present antigenically, however, the active enzyme dimer was undetectable.

\section{MATERIALS AND METHODS}

$p$-Nitrocatechol sulfate was obtained from Sigma Chemical Company (St. Louis, MO). Sepharose 4B was obtained from Pharmacia Fine Chemicals, Inc. (Piscataway, NJ). All other reagents were reagent grade. The enzymatic activity of ASA and the residual activity of the enzyme in immunoprecipitates towards the chromogenic substrate, nitrocatechol sulfate, were determined as previously described (18).

The soluble fraction of liver homogenates was prepared from three normal, one juvenile MLD, and three late infantile MLD livers (18). The purified normal liver ASA and its inactive subunits were prepared as previously described (19). Protein was determined according to the method of Lowry et al. (10). Polyacrylamide disc electrophoresis was performed as described previously (19). Preparative electrophorsis was carried out at pH 4.0 with a dual vertical polyacrylamide slab gel (Bio-Rad model 220) with $3 \mathrm{~mm}$ slab gel thickness and a constant amperage $(30 \mathrm{~mA} / \mathrm{slab})$ for $2 \mathrm{hr}$. The slab was scanned for protein bands at $280 \mathrm{~nm}$ by a scanning densitometer (Transidyne General, Ann Arbor, MI) and the acrylamide band containing the protein fraction isolated. This fraction was homogenized with a glass hand homogenizer, dispersed in saline, emulsified with an equal volume of the adjuvant, and used for immunization.

New Zealand white rabbits were each immunized with 1-1.5 mg of the various antigens in complete Freund's adjuvant (Difco) at multiple intradermal sites. For each antigen, at least two rabbits were immunized. Starting 10 days after immunization, the rabbits were bled weekly from the marginal ear vein. Antibody content of the individual bleedings, determined by quantitative precipitin tests, was found to be $1.5-2.5 \mathrm{mg} / \mathrm{ml}$. Antibody titer remained at this level for several months without the need for a booster injection. The antisera were pooled and the IgG fraction prepared by the method of Levy and Sober (9).

Specific insoluble immunoadsorbants were prepared by binding $150 \mathrm{mg}$ of the antisera IgG fraction to $5 \mathrm{~g}$ activated Sepharose 4B according to the method of Axen and associates (2). Double diffusion in agar gel was carried out as previously 
described (18). Immunoelectrophoresis was carried out in 0.05 $\mathrm{M}$ barbital buffer, $\mathrm{pH} 8.2$, using agarose coated sheets (Bioware, Inc., Wichita, KS) with a constant voltage of $6 \mathrm{~V} / \mathrm{cm}$ for $3.5 \mathrm{hr}$.

\section{RESULTS}

\section{ANTISERA TO ENZYMATICALLY ACTIVE ASA}

The antiserum obtained by immunizing rabbits with purified ASA from human liver, was designated as anti-ASA. When the purified ASA preparation was applied for electrophoresis at $\mathrm{pH}$ 8.5 , one band of protein with enzymatic activity was demon-

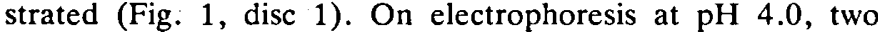
distinct activity bands (Fig. 1, disc 2) and four protein bands (Fig. 1, disc 3) were observed. The first band, faint on protein staining, exhibited the greatest enzymatic activity whereas the second band exhibited a large amount of protein and low enzyme activity. The quantitative interrelation between the two active forms varied in different preparations, however, both bands could always be visualized. The protein with low enzymatic activity, termed ASA(a), was separated by preparative electrophoresis at $\mathrm{pH} 4.0$, used as an antigen, and the antiserum obtained designated as anti-ASA(a).

Normal liver homogenate samples were applied, in duplicate, for electrophoresis at $\mathrm{pH} \mathrm{4.0.} \mathrm{One} \mathrm{disc} \mathrm{was} \mathrm{stained} \mathrm{for} \mathrm{protein}$ and the second disc placed on an agarose plate, $1 \%$ in phosphate-buffered saline (PBS) $(0.14 \mathrm{M} \mathrm{NaCl}$ in $0.01 \mathrm{M}$ phosphate buffer, pH 7.0) containing $2 \%$ of the anti-ASA(a). The results are shown in Figure 2. Two bands of immunoprecipitate corresponding to the two activity bands of purified ASA were observed. The anti-ASA(a) was shown to be specific to ASA as only the two active forms of the enzyme revealed detectable immunoprecipitate.

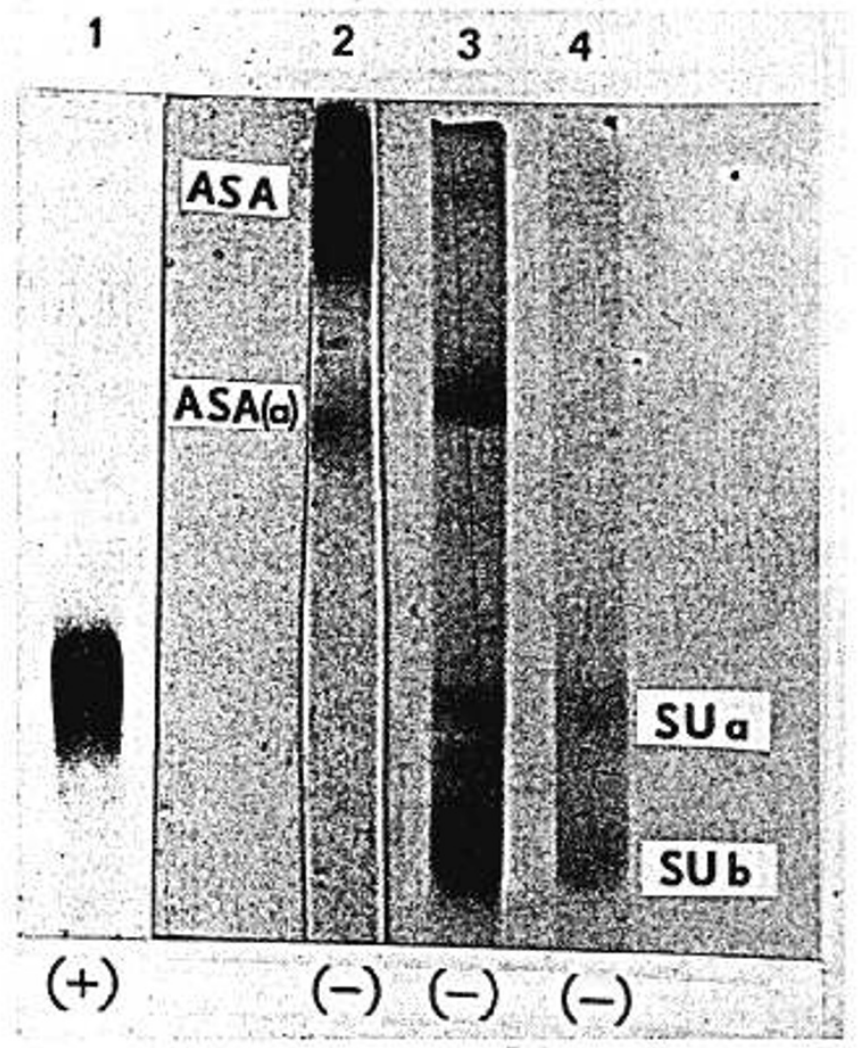

Fig. 1. Polyacrylamide gel electrophoresis of purified ASA. Disc 1: $\mathrm{pH} 8.5$, protein staining; disc $2: \mathrm{pH} 4.0$, activity pattern; disc $3: \mathrm{pH} 4.0$, protein staining; disc $4: \mathrm{pH} 4.0$, protein staining. Disc 4 depicts the enzyme after SDS treatment.

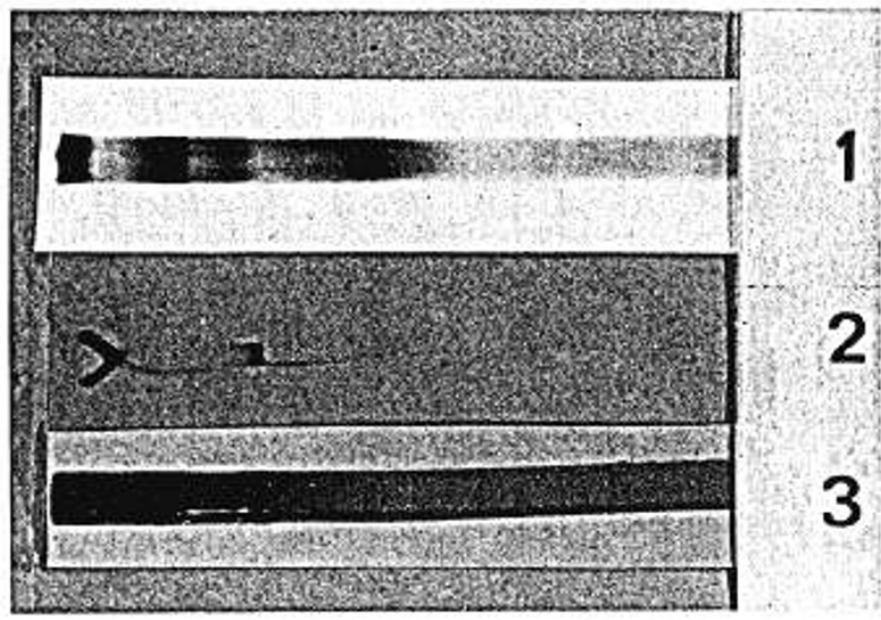

Fig. 2. Polyacrylamide gel electrophoresis of normal liver homogenates and purified ASA at $\mathrm{pH}$ 4.0. Disc 1: protein staining of normal liver homogenates; disc 3: activity pattern of purified ASA; plate 2: the immunoprecipitate obtained by applying a duplicate of disc 1 on an agarose plate containing anti-ASA(a) antibodies.

\section{IMMUNOLOGIC REACTIVITY OF NORMAL AND LATE INFANTILE} MLD LIVER HOMOGENATES

Three late infantile MLD liver homogenates and three normal liver homogenates were compared by double gel diffusion against the two antisera. Normal and MLD homogenates gave a precipitin line with anti-ASA (Fig. $3 A$ ). Only normal liver gave a precipitin line with anti-ASA(a) even when the protein concentration of the MLD samples was increased 10-fold (Fig. 3C). When both plates were stained for residual enzymatic activity, only the immunoprecipitate with the normal liver demonstrated activity (Fig. $3, B$ and $D$ ).

\section{ANTISERUM TO ENZYMATICALLY INACTIVE ASA SUBUNIT}

The inactive subunits were prepared from $15 \mathrm{mg}$ purified ASA by incubation in $1 \%$ SDS at room temperature, chromatographed on a Bio-Gel A $0.5-\mathrm{m}$ column and dialyzed as previously described (19).

Two distinct protein bands $\left(\mathrm{SU}_{\mathrm{a}}\right.$ and $\mathrm{SU}_{\mathrm{b}}$ ) were observed when this preparation was examined by electrophoresis at $\mathrm{pH}$ 4.0 (Fig. 1, disc 4 ). When the subunit preparation was examined by double gel diffusion (Fig. 4) and immunoelectrophoresis (Fig. 5), immunoprecipitates were observed with anti-ASA but not with anti-ASA(a). However, immunologic reactivity of one of the two subunits with anti-ASA(a) could be demonstrated by using the insoluble IgG fraction of the anti-ASA(a). Several attempts to separate the two distinct subunits by preparative acrylamide electrophoresis at $\mathrm{pH} 4.0$ to be used for immunization were unsuccessful. Based on this observation, the specific antisera towards one of the subunits was prepared using the following procédure: $300 \mathrm{mg}$ anti-ASA(a) IgG fraction were bound to $10 \mathrm{~g}$ activated Sepharose 4B. The immunoadsorbent was dispersed in $100 \mathrm{ml}$ of $0.1 \mathrm{M}$ sodium citrate-citric acid buffer, pH 3.8, containing $1 \%$ SDS, stirred for $1 \mathrm{hr}$ at $38^{\circ}$, and exhaustively washed with PBS. The washed immunoadsorbent was stirred with $10 \mathrm{mg}$ inactive subunits in $100 \mathrm{ml}$ PBS at room temperature for $2 \mathrm{hr}$. The unadsorbed fraction was separated and the adsorbent washed until the optical density of the effluent at $280 \mathrm{~nm}$ was lower than 0.01 . The adsorbed fraction was then eluted with $30 \mathrm{ml}$ of $0.1 \mathrm{M}$ sodium citrate-citric acid buffer containing $1 \%$ SDS at $38^{\circ}$. The eluate was dialyzed exhaustively against PBS and concentrated by negative pressure ultrafiltration. The protein content of this fraction was $4.4 \mathrm{mg}$. Electrophoresis revealed only one protein band corresponding to $\mathrm{SU}_{\mathrm{a}}$ of Figure 1, disc 4. This subunit preparation was used for immunization and the antiserum designated as anti-SUa. 
IMMUNOLOGIC COMPARISON OF ASA FROM NORMAL, LATE INFANTILE MLD AND JUVENILE MLD LIVER HOMOGENATE

The specificity of the three antisera (anti-ASA, antiASA(a), and anti-SU $\mathrm{a}_{\mathrm{a}}$ was documented by examining purified ASA and normal liver homogenate against each of the antisera by double gel diffusion and immunoelectrophoresis. Identical precipitin

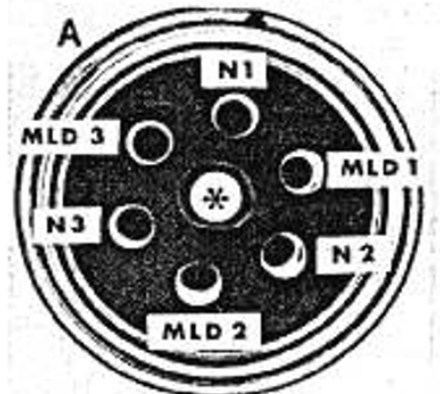

* Anei ASA

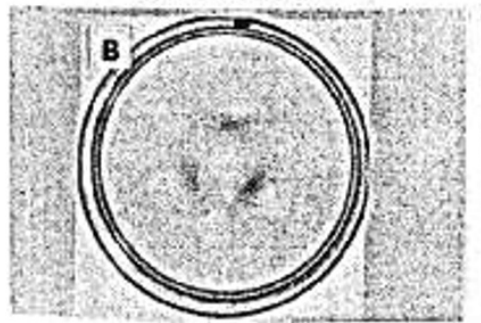

Fig. 3. Double gel diffusion. $\mathrm{N}_{1}, \mathrm{~N}_{2}$, and $\mathrm{N}_{3}$ : normal liver homogenates; $M L D_{1}, M L D_{2}$, and $M_{L} D_{3}$ : liver homogenates from patients with late infantile MLD. Plates A and B: anti-ASA antiserum has been applied to the central well (A shows the immunoprecipitin lines and $B$ depicts the residual enzymatic activity). Plates C and D: anti-ASA(a) antiserum has been applied to the central well.

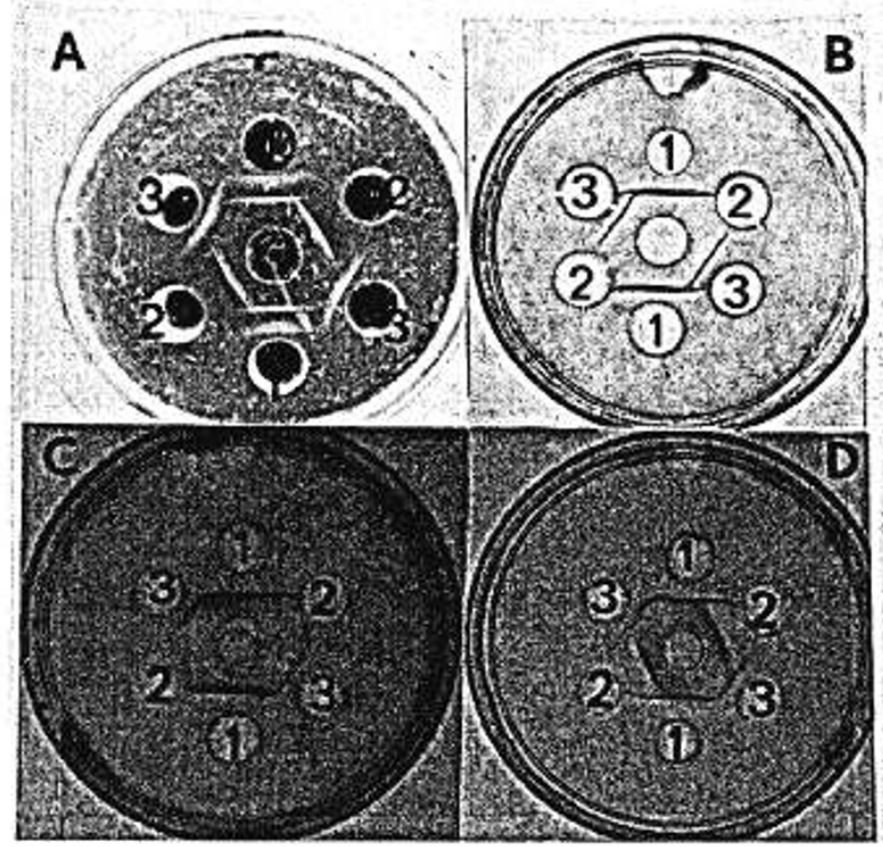

Fig. 4. The immunoprecipitin line obtained by double gel diffusion of normal liver homogenates (plate $A$ ), the inactive subunits of purified ASA (plate B), late infantile MLD liver homogenates (plate C), and juvenile MLD liver homogenates (plate $D$ ). Various antiserm have been applied to the following peripheral wells: Well 1: anti-ASA; well 2: antiASA(a); well 3: anti-SUa.

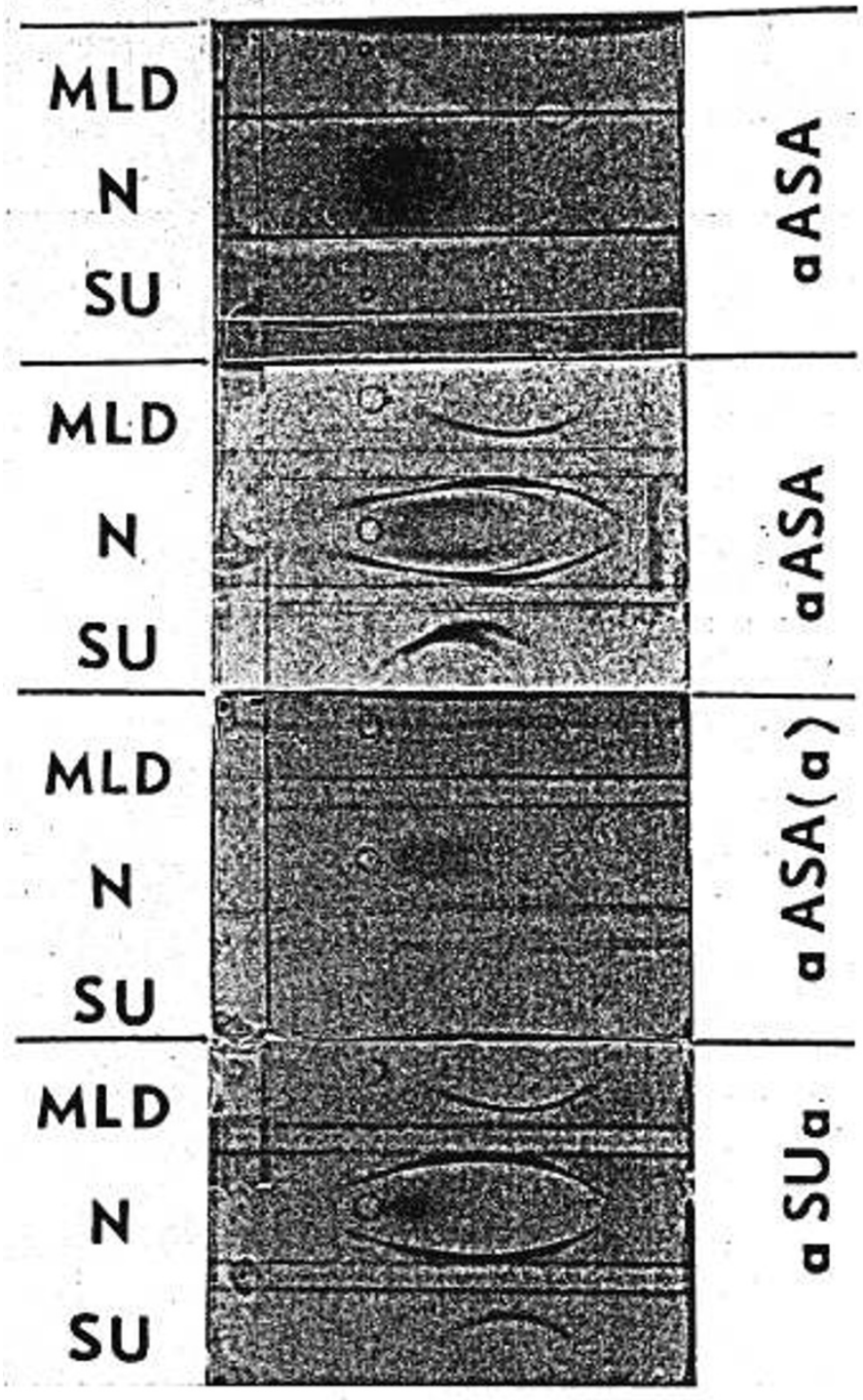

Fig. 5. Immunoelectrophoresis of late infantile MLD liver homogenates (MLD), normal liver homogenates $(\mathrm{N})$, and the inactive subunits of purified ASA (SU). Antisera (aASA: anti-ASA; aASA(a): antiASA(a); and aSU $\mathrm{a}_{\mathrm{a}}$ anti-SU $\mathrm{a}_{\mathrm{a}}$ in the slots of each set are designated on the right side. In the upper set, the residual enzymatic activity of the immunoprecipitates is depicted.

lines were obtained with both antigen preparations. These three antisera were examined as to their immunologic reactivity with normal, late infantile MLD, juvenile MLD livers, and the inactive subunit preparations. The results are shown in Figure 4. The normal liver gave a double line with anti-ASA (Fig. 4A). Only one line was observed when freshly prepared homogenates were studied: The second line became more prominent after prolonged storage or repeated freezing and thawing of the homogenate sample. A similar phenomenon was also shown with purified ASA preparations. The line appearing after repeated freezing and thawing coincided with the precipitin line obtained with anti-SU $\mathrm{S}_{\mathrm{a}}$ and did not exhibit enzymatic activity: The line which exhibited enzymatic activity formed a "double spur" with the precipitin line of anti-ASA(a), indicating the presence of antibodies toward both common and specific antigenic determinants. The precipitin line of the inactive subunits with anti-ASA revealed a spur formation with the line against anti-SU $U_{\mathrm{a}}$. No precipitate could be visualized between the inactive subunits preparation and anti-ASA(a), even after staining (Fig. $4 B$ ). A similar precipitate pattern was observed when the 
liver homogenate from the late infantile MLD patient was examined with the exception that no spur formation was present (Fig. 4C). The liver homogenate from the patient with the juvenile MLD revealed a single identical precipitin line with anti-ASA and anti-SU $\mathrm{a}_{\mathrm{a}}$ In contrast to the subunit preparation and late infantile MLD, a precipitin line was also observed when juvenile MLD was studied with anti-ASA(a) (Fig. 4D).

IMMUNOELECTROPHORETIC COMPARISON OF VARIOUS ANTIGENICALLY CROSS-REACTING FORMS OF ASA IN NORMAL AND MLD LIVER HOMOGENATES

Immunoelectrophoresis was performed in an attempt to demonstrate the various immunologically cross-reacting forms of ASA and its subunits. The results are shown in Figure 5. The normal liver homogenate gave three precipitin arcs with antiASA. One could be demonstrated with all of the antisera and coincided with the precipitate with enzymatic activity. The two other precipitin arcs did not exhibit enzymatic activity and were seen in the inactive subunit preparation. Only one of these subunit arcs was demonstrated in the late infantile MLD sample. The same subunit arc was seen with anti-SUa.

The difference between the antigenically cross-reacting material in the two clinical forms of MLD is shown in Figure 6. With anti-ASA, the juvenile MLD sample gave two precipitin arcs of which only one could be demonstrated in late infantile MLD. The arc that was obtained in both forms of the disease was

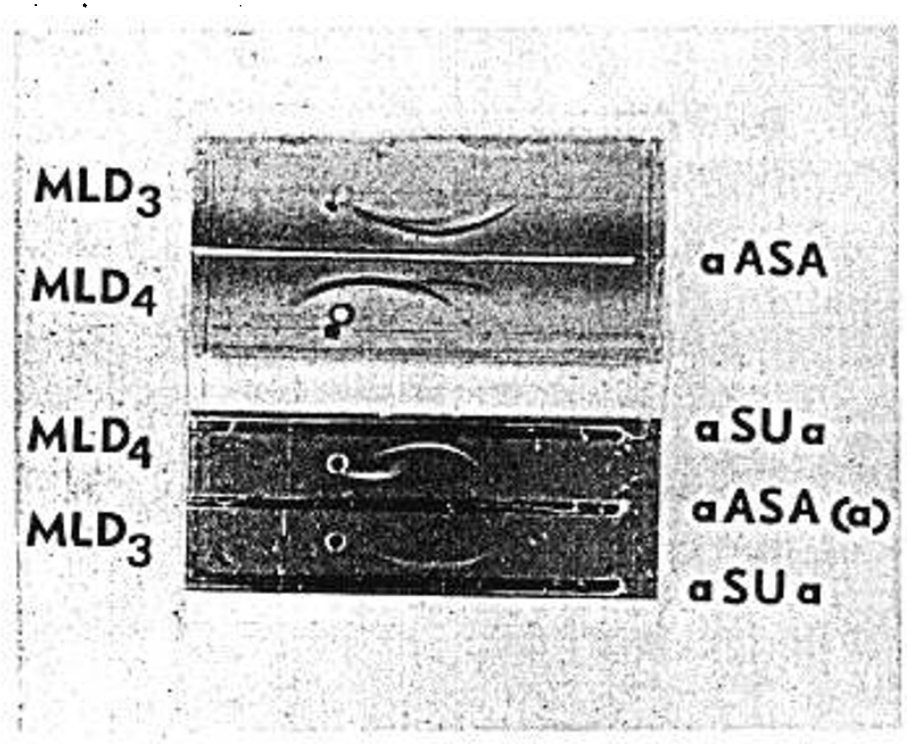

Fig. 6. Immunoelectrophoresis of late infantile MLD liver homogenates $\left(\mathrm{MLD}_{3}\right)$ and juvenile $\mathrm{MLD}$ liver homogenates $\left(\mathrm{MLD}_{4}\right)$ with the three antisera as in Figure 5. identical to that demonstrated with anti-SUa. Only the juvenile MLD sample revealed an immunoprecipitate with anti-ASA(a).

\section{DISCUSSION}

In the present study, ASA from normal late infantile MLD and juvenile MLD livers were compared as to their immunologic cross-reactivity with three antisera preparations: purified ASA (anti-ASA); one to an ASA component with a decreased enzymatic activity (anti-ASA(a)); and a third against one of the inactive subunits (anti-SU $U_{\mathrm{a}}$ ). The monospecificity of the antiASA(a) is clearly evident as it reacted only with the active enzyme forms. The anti-SU $U_{a}$ reacted with only one of the two inactive subunits and with the active enzyme, whereas anti-ASA reacted with both active enzyme forms as well as with both inactive subunits. Similar results were obtained with purified ASA as with crude liver homogenates. None of the liver proteins besides the four ASA components was immunologically reactive with the three antisera. Therefore, if ASA is composed of two distinct subunits, all three antisera prepared should be regarded as monospecific to ASA and its components. Draper et al. (5) have demonstrated that a highly purified major ASA from human liver could be dissociated by denaturation in SDS, $6 \mathrm{M}$ guanidine and in $6 \mathrm{M}$ urea into two distinct subunit components of 53,000 and $66,000 \mathrm{~mol}$ wt. One of these subunits $\left(\mathrm{SU}_{\mathrm{a}}\right)$ was purified by us, whereas the attempts to purify $S U_{b}$ in a sufficient amount for immunization were unsuccessful.

The immunologic reactivity of the three antisera obtained (anti-ASA, anti-ASA(a) and anti-SU ${ }_{a}$ ) with the various liver homogenate preparations and with ASA subunits is summarized in Table 1. These findings indicate that late infantile MLD and the juvenile form of MLD represent different molecular defects. Previous studies have demonstrated antigenic cross-reacting material in both late infantile and juvenile forms of MLD (12, $16,20)$. We have previously shown that this mutant protein is enzymatically inactive in liver homogenates from both entities (18). A mutational event leading to an inactive but immunologically cross-reacting material has been designated as a CRM+ mutant whereas a mutation leading to missing activity as well as no immunologically detectable protein has been termed a CRM - mutation in microbial genetics (21). Both CRM+ and CRM - human mutations have been reported even within the same clinical entity (3). Using the above classification, the late infantile MLD is CRM - for all active forms of ASA but CRM+ for only one of the inactive subunits (Figs. $4 C$ and 5). In contrast, the juvenile form of MLD is CRM+ for both inactive subunits and for ASA(a) (Figs. 4D and 6).

The observation of a protein with an enzymatic activity, ASA(a), that differs in its electrophoretic mobility (Fig. 1) but shares antigenic determinants (Fig. 2) with the major active form of ASA raises interesting questions. It is unlikely that this is an artifact of the purification as it was shown both in freshly prepared liver homogenates and in purified enzyme preparations (19). In addition, it is also unlikely that ASA(a) is a partially denatured form of ASA as they demonstrated immunologic

Table 1. Immunologic reactivity of ASA from normal and MLD liver homogenates with various antisera ${ }^{1}$

\begin{tabular}{|c|c|c|c|c|c|c|c|c|c|}
\hline$\because$ & \multicolumn{3}{|c|}{ Anti ASA } & \multicolumn{3}{|c|}{ Anti ASA(a) } & \multicolumn{3}{|c|}{ Anti-SU ${ }_{a}$} \\
\hline Late infantile MLD & + & - & 1 & - & - & - & + & - & 1 \\
\hline Juvenile MLD & + & - & 2 & + & - & 1 & + & - & 1 \\
\hline Enzymatically inactive ASA subunits & + & - & 2 & - & - & - & + & - & 1 \\
\hline
\end{tabular}

${ }^{1}$ DGD: presence (+) or absence $(-)$ of a precipitin line by double gel diffusion; IEP active: precipitin arcs with residual ASA activity revealed by immunoelectrophoresis at pH 8.2; IEP inactive: precipitin arcs with no enzymatic activity by electrophoresis at pH 8.2.

${ }^{2}$ One arc by electrophoresis at $\mathrm{pH} 8.2$ and two at $\mathrm{pH} 4.0$. 
dissimilarities that could be explained only by a selective loss of some antigenic determinants. One possible explanation might be that $\mathrm{ASA}(\mathrm{a})$ is a different isoenzyme with very similar size and charge to the major active ASA and both are copurified by our purification procedure (19). It is shown that both active enzyme forms share antigenic determinants (Figs. 2 and 4), indicating either a common subunit or structural similarity. The most likely explanation of the present data is therefore that the major active ASA isozyme is composed of two subunits, $\mathrm{SU}_{\mathrm{a}}$ and $S U_{b}$, that differ antigenically. The minor active form ASA(a) might, therefore, be a dimer of one subunit, the $\mathrm{SU}_{\mathrm{a}}$.

Consistent with this model, our antiserum against the purified enzyme anti-ASA is a polyvalent antiserum. Antibodies directed against antigenic determinants in ASA and ASA(a) and their respective monomeric subunits, $S U_{a}$ and $S U_{b}$, are all present in this antiserum preparation. The denatured subunits, $\mathrm{SU}_{\mathrm{a}}$ and $S U_{b}$, were produced either while homogenizing the antigen with the adjuvant or in the immunized rabbit. Therefore, the antiASA contains antibodies against determinants in the denatured subunits and reveals precipitin lines with the subunit preparations (Fig. $4 C$ ) as well as with the subunits present in a liver homogenate after freezing and thawing (Fig. $4 A$ ). The antiserum prepared towards ASA(a), purified by preparative electrophoresis, is monospecific and cross reacts with the active isoenzyme forms, ASA and ASA(a) (Fig. 2). The denatured subunits of $\mathrm{ASA}$ (a) are $\mathrm{SU}_{\mathrm{a}}$ in which most of the antigenic determinants are altered to such an extent that no immunologic cross-reactivity could be observed by double gel diffusion with anti-ASA(a) (Fig. 4). The insoluble anti-ASA(a) immunoadsorbent adsorbed $\mathrm{SU}_{\mathrm{a}}$, either by the few antigenic determinants preserved or via low affinity antibodies.

In the immunoelectrophoresis and disc electrophoresis, the two active isoenzyme forms are indistinguishable in their mobility at $\mathrm{pH} 8.2$ and $\mathrm{pH} \mathrm{8.5,} \mathrm{respectively} \mathrm{(Fig.} \mathrm{1,} \mathrm{disc} \mathrm{1,} \mathrm{and} \mathrm{Fig.}$ $5)$. The three antisera reacted with the active isoenzymes revealing an identical precipitin arc with residual enzymatic activity (Fig. 5).

If one accepts our premise that the two isoenzymes share a common subunit, the antigenic differences in late infantile MLD and the juvenile form of MLD are explainable as follows. The mutation in late infantile MLD occurred in the common subunit, $S U_{a}$, leading to the absence of both active enzyme forms. The other subunit, $\mathrm{SU}_{\mathrm{b}}$, is preserved in vivo only when it is present in the dimer. In juvenile MLD, a point mutation in the same subunit, $\mathrm{SU}_{\mathrm{a}}$, leads to the production of a labile form of both isoenzymes. The residual activity of the mutant is lost in the preparation of the homogenate. Therefore, the two subunits are $\mathrm{CRM}+$. This model of two isoenzymes might well explain the differences in the clinical manisfestations, the observation of different amounts of storage material at autopsy (8), and the finding of different sulfatide accumulation in fibroblasts (16).

\section{CONCLUSION}

Antisera were prepared against arylsulfatase A purified from human liver. The antisera were prepared against a minor component with enzymatic activity isolated by preparative acrylamide electrophoresis at $\mathrm{pH} 4.0$ and against one of the two inactive subunits of the enzyme. When normal liver homogenates were examined by immunoelectrophoresis with these three antisera, the active dimer could be distinguished from the two inactive subunits. In liver homogenates from late infantile metachromatic leukodystrophy, the active dimer as well as one of the subunits was absent. In contrast, in the juvenile form of the disease both inactive subunits were immunologically detectable whereas the active dimer could not be detected.

\section{REFERENCES AND NOTES}

1. Austin, J., Armstrong, D., and Shearer, L.: Metachromatic leukodystrophy. $V$. The nature and significance of low sulfatase activity: a controlled study of brain, liver and kidney in four patients with metachromatic leukodystrophy. Arch. Neurol., 13: 593 (1965).

2. Axen, R., Porath, J., and Ernback, S.: Chemical coupling of peptides and proteins to polysaccharides by means of cyanogen halides. Nature, 214: 1302 (1967)

3. Boyer, S. H., Siggers, D. S., and Krueger, L. J.: Caveat to protein replacement therapy for genetic disease: immunological implications of accurate molecular diagnosis. Lancet, II: 654 (1973).

4. Dodgson, K. S., Spencer, B., and Wynn, C. H.: Studies of sulphatases. 12. The arylsulphatases of human tissues. Biochem. J., 62: 500 (1956).

5. Draper, R. K., Stout, R. E. L., and Edmond, J.: Studies with arylsulfatase A from human liver. Trans. Amer. Soc. Neurochem., 6: 136 (1975)

6. Goldstone, A., Konecny, P., and Koenig, H.: Lysosomal hydrolases: conversion of acidic to basic forms by neuraminidase. FEBS Lett., 13: 68 (1971).

7. Harzer, K., Stinshoff, K., Mraz, W., and Jatzkewitz, H.: The patterns of arylsulphatases $\mathbf{A}$ and $\mathbf{B}$ in human normal and metachromatic leukodystrophy tissues and their relationship to the cerebroside sulphatase activity. $J$. Neurochem., 20: 279 (1973).

8. Jatzkewitz, H., Pilz, H., and Holländer, H.: Biochemische und vergleichende histochemische Untersuchungen in umschriebenen Gebieten das Gehirns bei Fällen on adulter und infantiler metachromatischer leukodystrophie. Acta Neuropathol., 4: 75 (1964).

9. Levy, H. B., and Sober, H. A.: A simple chromatographic method for preparation of gammaglobulin. Proc. Soc. Exp. Biol. Med., 103: 250 (1960).

10. Lowry, O. H., Rosebrough, N. J., Farr, A. L., and Randall, R. J.: Protein measurement with the Folin phenol reagent. J. Biol. Chem., 193: 265 (1951).

11. Mehl, E., and Jatzkewitz, H.: Cerebroside 3-sulfate as a physiological substrate of arylsulfatase A. Biochim. Biophys. Acta, 151: 619 (1968).

12. Neuwelt, E., Stumpf, D., Austin, J., and Kohler, P.: A monospecific antibody to human sulfatase $A$ preparation, characterization and significance. Biochim. Biophys. Acta, 236: 333 (1971).

13. Neuwelt, E., Stumpf, D., Austin, J., and Kohler, P.: Recent advances in metachromatic leukodystrophy. Advan. Exp. Med. Biol., 19: 415 (1972).

14. Nichol, L. W., and Roy, A. B.: The sulfatase of ox liver. X. Some observations on the intermolecular bonding in sulfatase A. Biochemistry, 5: 1379 (1966)

15. Nicholls, R. G., and Roy, A. B.: Arylsulfatases. In: P. D. Boyer: The Enzymes, Vol. 5, p. 21 (Academic Press, New York, 1971).

16. Porter, M., Fluharty, A., Trammell, J., and Kihara, H.: A correlation of intraellular cerebroside sulfatase activity in fibroblasts. Biochem. Biophys. Res. Commun., 44: 660 (1971).

17. Roy, A. B., and Jerfy, A.: The sulphatase of ox liver. XIV. The subunit structure of sulphatase A. Biochim. Biophys. Acta, 207: 156 (1970).

18. Shapira, E., and Nadler, H. L.: The nature of the residual arylsulfatase activity in metachromatic leukodystrophy. J. Pediat., 86: 881 (1975).

19. Shapira, E., and Nadler, H. L.: Purification and some properties of soluble human liver arylsulfatases. Arch. Biochem. Biophys., 170: 179 (1975).

20. Stumpf,' D. A., Neuwelt, E., Austin, J., and Kohler, P.: Metachromatic leukodystrophy (MLD), immunological studies of the abnormal sulfatase A. Arch. Neurol., 25: 427 (1971).

21. Suskind, S. R.: Properties of a protein antigenically related to tryptophan synthetase in Neurospora crassa. J. Bacteriol., 74: 308 (1957).

22. ASA(a), the arylsulfatase form with residual enzymatic activity; $S_{\mathrm{a}}$ and $\mathrm{SU}_{\mathrm{b}}$, the two inactive ASA subunits; anti- preceding the antigen designation, the various antisera.

23. We are indebted to Mrs. D. J. Goins and Miss L. A. Posner for their skilled technical assistance.

24. This study was supported by grants from the National Institutes of Health (HD 04252), The National Foundation-March of Dimes, The Kroc Foundation, and The Spastic Paralysis Research Foundation.

25. Emmanuel Shapira is on leave from the Hadassah University Hospital, Hebrew University, Jerusalem, Israel.

26. Henry L. Nadler is the Irene Heinz Given and John La Porte Given Research Professor of Pediatrics.

27. Requests for reprints should be addressed to: Henry L. Nadler, M.D., Children's Memorial Hospital, 2300 Children's Plaza, Chicago, IL 60614 (USA).

28. Received for publication February 28, 1977.

29. Accepted for publication June 10, 1977. 